Objectives: To describe the clinical features and outcomes of septic spondylodiscitis and to identify factors associated with an unfavourable clinical outcome.

Methods: Retrospective study including 107 patients followed up in our department between 1999 and 2019. Clinical, radiological and microbiolog ical data were collected.

We divided patients into two groups: patients with unfavourable clinical outcome (defined as death, drug toxicity, neurological complication, sepsis or persistent pain) (group 1) and patients with a favourable one (Group 2).

Results: We included 107 patients (49 women and 58 men), with a mean age of 55 years old [16 - 86]. The median delay of consultation was 3 months. Predisposing factors were found in 59 patients (55.1\%). Inflammatory back pain was seen in $78.5 \%$ of cases. Neurologic deficiency was noticed in $16.82 \%$ of cases: motor deficit in $1.8 \%$ of cases, spinal cord compression in $1.8 \%$ of cases and Cauda equina syndrome in $2.8 \%$ of cases. An inflammatory biological syndrome was found in $90.6 \%$ of cases. The lumbar spine was involved in $55 \%$. The spondylitis was multifocal in $19.6 \%$ and multi-stage in $15.8 \%$ of cases. CT and Spinal MRI was performed respectively in $60 \%$ and $78.8 \%$ of cases and showed paravertebral abscess in $63.5 \%$, epiduritis in $54.2 \%$, intra-discal abscess in $4.67 \%$, spinal cord compression 9.3\%, and vertebral ostelysis in $8.4 \%$ of cases. The causative microorganism was mycobacterium tuberculosis in $59.8 \%$, brucella in $20.56 \%$, and pyogenic germs in $16.8 \%$ of cases. $34.5 \%$ of patients had an unfavourable clinical outcome: persistent pain was noticed in $18.7 \%$,drug toxicity occurred in $13 \%$ of cases, neurological complication occurred in $10.2 \%$ of cases, sepsis occurred $3.7 \%$ of cases and $3.7 \%$ of patients were dead.

In the group1 the frequency of diabetes, impairment of the general state and clinical evidence of neurological impairment at presentation was higher, but with no statistically significant difference. Similarly, the presence of paravertebral abscess, epiduritis or spinal cord compression was slightly more frequent, with no significant difference.

There was no statically significant difference in the age $(p=0.15)$, the localisation and the causative microorganism ( $p=0.68)$.

Conclusion: Spondylodiscitis is a rare but serious condition that leads to significant long-term morbidity. In our study, unfavourable clinical outcomes was found in the presence of diabetes, neurological impairement at presentation and the presence of paravertebral abscess, epiduritis or spinal cord compression in MRI but with no statically significant difference.

Disclosure of Interests: None declared

DOI: 10.1136/annrheumdis-2019-eular.8154

\section{AB1248 MALHEUR PROJECT CONFIRMS SPECIFIC CHARACTERISTICS OF MALIGNANCIES IN PATIENTS WITH RHEUMATIC AND MUSCULOSKELETAL DISEASES}

Karolina Benesova, Maria Czaja, Regina Max, Hanns-Martin Lorenz. University Hospital Heidelberg, Department of Medicine V, Division of Rheumatology, Heidelberg, Germany

Background: Both rheumatic and musculoskeletal diseases (RMDs) and malignancies are frequent in the population and the probability of simultaneous occurrence in one individual is accordingly high. However, the interrelation of malignancies in patients with RMDs is still a blind spot in rheumatology. Since both disorders present two extremes of a dysregulated immune response, their interdependencies are undoubtedly complex. There is very little reliable data on cancer and relapse risk due to a certain disease entity as well as particular disease modifying therapies.

Objectives: The MalheuR project is a registry-based study that has been initiated at the university hospital Heidelberg, Germany to close this gap. Methods: In three subregistries, we address the specific situation of patients suffering from concomitant RMD and malignancy (RheuMal registry), paraneoplastic rheumatic disorders due to a malignancy (ParaRheuMa registry) and rheumatic immune-related adverse events (irAE) due to cancer immunotherapy (TRheuma registry). Herein, we present first data from RheuMal registry.

Results: We analyzed 55 patients with concomitant RMDs and malignancies. The mean onset of the RMD was 58.9 years in females $(n=33)$ and 54.2 years in males $(n=22)$, the mean onset of the malignancy was 59.9 years and 56.3 years respectively. In patients first suffering from a RMD ( $n=38)$, the average time to malignancy diagnosis was 10.3 years [1-33 years]. Only 13 patients were diagnosed with a malignancy before RMD. 4 were diagnosed for both disorders simultaneously. Solid tumors were present in 54\%, hematologic neoplasms in $17 \%$, non-melanoma skin cancer in $21 \%$ and melanoma in $8 \% .13$ patients suffered from more than one neoplasm with 1 patient showing a secondary neoplasm, 4 patients with recurring skin cancer and 4 with additional benign neoplasms. Out of 6 patients pre-treated with cyclophosphamide, lymphoma bladder and prostate cancer were diagnosed in one case each. 16 patients were pre-treated with TNFa-inhibitors, 2 cases of melanoma, 4 cases of non-melanoma skin cancer and 2 cases of hematologic neoplasms occurred. Among 7 patients with azathioprine intake, two developed non-melanoma skin cancer, but no melanoma was diagnosed.

A comparison with the German cancer registry of the Robert Koch Institute suggests an earlier onset of gender specific cancers in our patients with mean onset of breast cancer $(n=5) 5.2$ years and prostate cancer $(n=8) 7.0$ years earlier. Furthermore, in males with RMDs melanoma occurred 8.4 years earlier $(n=5)$, but squamous cell skin cancer was diagnosed 12.2 years later than in the reference cohort.

Conclusion: Overall, our data show specific characteristics of malignancies in RMD patients suggesting a different cancer epidemiology compared to overall population.

Disclosure of Interests: Karolina Benesova Grant/research support from: Scientific Projects (IIT) I'm involved in as an investigator have been supported, but not initiated or designed by Abbvie and Novartis, Consultant for: Advisory board member on early disease detection for Novartis, Maria Czaja: None declared, Regina Max: None declared, Hanns-Martin Lorenz: None declared

DOI: 10.1136/annrheumdis-2019-eular.4188

\section{AB1249 HOSPITAL ADMISSIONS IN PATIENTS WITH CHRONIC RHEUMATIC DISEASESRECEIVING ADALIMUMAB. DESCRIPTIVE STUDY OF A COHORT}

Boris Anthony Blanco Cáceres, Fernando Perez-Ruiz, Paula García Escudero, Marta González Fernández. Hospital Universitario Cruces, Rheumatology, Barakaldo, Spain

Background: Patients with chronic rheumatic diseases (CRD) treated with biological DMARDs may increase the risk of complications and hospitalizations for serious adverse reactions (SAR). Concomitant treatment with conventional DMARDs and corticosteroids may increase the risk of complications.

Objectives: To describe the prevalence and characteristics of hospital admissions among patients with CRD who are currently receiving Adalimumab (ADL) in a hospital setting.

Methods: Cross-sectional, retrospective, unicentric study. Data obtained from a cross-sectional study to determine levels of $A D L$ and antidrug antibodies in patients currently receiving $A D L$. We clustered the income in SAR and income not related to bDMARD, describe the clinical and therapeutic data. Groups were compared using Student's t-test and Chisquared test.

Results: We included 103 patients on ADL treatment, $54.4 \%$ (56) men, $45.6 \%$ (47) women. The mean age was 54.6 years (SD \pm 13.04 ). 38.8\% diagnosed of rheumatoid arthritis, $42.7 \%$ spondyloarthritis, $15.5 \%$ psoriatic arthritis and $2.9 \%$ juvenile idiopathic arthritis.

$33 \%$ (34) had at least one income, mean 2.29 SD \pm 2.93 (1-17). Within this group, the mean age was 62.3 years $(S D \pm 9.93)$, the mean time of exposure to ADL was 98.9 months SD \pm 43.68 (3.48-151.49). $44.1 \%$ (15) had a standard dose of ADL and $55.9 \%$ (19) had a minimum effective dose. 55.9\% (19) also received scDMARD and $47.1 \%$ (16) oral corticosteroids with a mean dose of prednisone of $3.4 \mathrm{mg}$ (SD \pm 4.6 ). 78 incomes were produced, $7(9 \%)$ by SAR and $71(91 \%)$ not related to bDMARD. The average income per SAR was $0.50 \mathrm{SD} \pm 1.48$

The mean time of exposure to ADL in the group of patients with SAR was 101.97 months $S D \pm 33.8$, in contrast to patients without hospital admissions that was 65.02 months $S D \pm 50.17$ ( $p<0.01)$. Patients without incomes received a mean daily dose of prednisone of $2.45 \mathrm{mg} / \mathrm{day}$ $\mathrm{SD} \pm 3.71$ while those with SAR admission of $4.58 \mathrm{mg} /$ day $\mathrm{SD} \pm 4.00$ (p <0.05).

Conclusion: $33 \%$ (34) of patients had at least one admission, they were older (62.3 years SD \pm 9.93$)$ than those without incomes. The mean of income was 2.29 SD \pm 2.93 (1-17). $55.9 \%$ of patients also had a scDMARD prescribed and $47.1 \%$ oral corticosteroids. $9 \%$ of the admissions were by SAR with an average ADL exposure of 101.97 months $\mathrm{SD} \pm 33.8$ compared to patients without hospital admissions, mean 65.02 months SD \pm 50.17 with statistically differences $(p<0.01)$. In addition, dif ferences were found regarding the dose of prednisone, patients with admissions by SAR received $4.58 \mathrm{mg} /$ day $S D \pm 4.00$ unlike those without incomes who received $2.45 \mathrm{mg} /$ day $S D \pm 3.71$ ( $p>0.05)$.

Weakness of the study is a selection bias since we include the data of patients with current ADL treatment, losing information of those in whom 